\title{
AVALIAÇÃO DAS CARACTERÍSTICAS HIDRÁULICAS DO TUBO PERFURADO SANTAPE ${ }^{1}$
}

\author{
Albanise Barbosa Marinho², José Dantas Neto ${ }^{3}$, Carlos Alberto Vieira de Azevedo ${ }^{3}$ \\ \& Hamilton Medeiros de Azevedo ${ }^{4}$
}

\begin{abstract}
RESUMO
Foram determinadas as características hidráulicas do Santape, que trata-se de um tubo perfurado flexível de polietileno linear de baixa densidade, com orifícios a raio laser. Avaliou-se a relação vazão-pressão, o coeficiente de variação de fabricação dos orifícios, o diâmetro interno do tubo e a ruptura de sua parede em função da variação de pressão, o alcance e a altura do jato da água, e a vazão em função do comprimento do tubo e da pressão. Os emissores apresentaram valor médio de coeficiente de variação de fabricação de $4,10 \%$ e regime turbulento $(x=0,5646)$. Verificou-se aumento do diâmetro interno do tubo em função de incrementos na pressão e a ruptura da parede do tubo ocorreu na pressão de $169,30 \mathrm{kPa}$. A vazão em função do comprimento variou de 11,25 a 7,09 $\mathrm{L} \mathrm{h}^{-1} \mathrm{~m}^{-1}$, dados obtidos experimentalmente para comprimentos de 10 a $200 \mathrm{~m}$ e pressão de $80 \mathrm{kPa}$.
\end{abstract}

Palavras-chave: irrigação localizada, vazão, hidráulica

\section{EVALUATION OF HYDRAULIC CHARACTERISTICS OF THE SANTAPE PERFORATED TUBE}

\begin{abstract}
The hydraulic characteristics of the Santape were determined, which is a perforated flexible polyethylene linear tube, of low density, with orifices made by laser rays. The discharge-pressure relationship, the manufacturing variation coefficient of the orifices, the inner diameters of tube and its wall rupture as a function of pressure variation, the reach and height of the water jet, and the discharge as a function of the tube length were evaluated. The emitters presented mean value for the manufacturing variation coefficient equal to $4.10 \%$ and a turbulent regime (discharge exponent of 0.5646). An increase in the inner diameter of the tube was verified as the pressure increased and the rupture of the wall occurred at $169.30 \mathrm{kPa}$ pressure. The discharge as a function of the tube length varied from 11.25 to $7.09 \mathrm{~L} \mathrm{~h}^{-1} \mathrm{~m}^{-1}$, data obtained experimentally for tube lengths from 10 to $200 \mathrm{~m}$ and pressure equal to $80 \mathrm{kPa}$.
\end{abstract}

Key words: localized irrigation, discharge, hydraulic

\section{INTRODUÇÃO}

O sistema de irrigação localizada diversifica-se quanto às características hidráulicas de vazão e distribuição de água de cada emissor, vazão na lateral, perda de carga etc.; assim, os métodos de irrigação localizada são divididos por gotejamento, microaspersão, xique-xique e tubos perfurados.

O funcionamento hidráulico dos tubos de polietileno perfurados assemelha-se a gotejadores tipo orifício, porém com a particularidade de que sua seção de saída aumenta com a

\footnotetext{
${ }^{1}$ Trabalho extraído da dissertação de Mestrado do primeiro autor apresentada à UFPB

${ }^{2}$ Eng. Agrícola, M.Sc. Engenharia Agrícola - Irrigação e Drenagem, DEAg/CCT/UFPB

${ }^{3}$ Prof. Adjunto, Doutor, DEAg/CCT/UFPB, Av. Aprígio Veloso 882, Bodocongó, CEP 58109 - 970, Campina Grande, PB, Fone: (083) 310 1318, Fax: (083)310 1185.E-mail: zedantas@ deag.ufpb.br, cazevedo@deag.ufpb.br

${ }^{4}$ Prof. Titular, M.Sc., DEAg/CCT/UFPB
} 
pressão em seu interior, em função da elasticidade de sua parede. Eles podem gotejar em baixas pressões; todavia, se a pressão for inferior a $100 \mathrm{kPa}$, as gotas se convertem em pequenos jatos contínuos (López et al., 1992).

Entre os tubos perfurados disponíveis no mercado nacional, encontra-se o Santape, de polietileno linear de baixa densidade, com matéria-prima virgem, o qual possui microfuros feitos a raio laser que, por sua vez, distribuem a água em jatos bem finos, em forma de spray e trabalha com uma pressão de serviço de 20 a $80 \mathrm{kPa}$.

Os emissores em um sistema de irrigação localizada devem apresentar descarga uniforme e constante, suficiente abertura para não provocar entupimentos e baixo custo, robustez e homogeneidade (Vermeiren \& Jobling, 1980).

Segundo von Bernuth \& Solomon (1986), Abreu et al. (1987) e Keller \& Bliesner (1990) o fluxo de um emissor é caracterizado por uma função potencial, em que a vazão se relaciona diretamente com a carga hidráulica atuante na entrada do emissor $\left(\mathrm{q}=\mathrm{k}_{\mathrm{d}} \mathrm{H}^{\mathrm{x}}\right)$. A constante $k_{d}$ inclui, no seu valor, os fatores relacionados à fabricação do emissor como, por exemplo, a área da seção transversal dos emissores tipo orifício; portanto, quando se consideram as variações de vazão do emissor devido à fabricação, deve-se referir, sempre, ao valor de $k_{d}$; em contrapartida, o expoente $x$ descreve como a vazão varia com a pressão e seu valor normalmente está entre zero e um, dependendo do regime de escoamento, determinado pelo número de Reynolds (Bralts et al., 1981).

Andrade (1990) e Bonomo et al. (1998) avaliando o tubo perfurado de polietileno flexível, obtiveram valores de $x$ iguais a 0,5491 e 0,50, indicando o regime de escoamento como turbulento. Para o tubo gotejador Queen Gil, Testezlaf \& Campioni (1993) encontraram $x=0,625$. Frizzone et al. (1998) avaliando as características hidráulicas do tubo gotejador RainTape TPC, encontraram o valor de $x$ como 0,4563 , ambos caracterizados como escoamento turbulento.

Segundo Vermeiren \& Jobling (1980) o coeficiente de variação de fabricação é o parâmetro usado para medir a variação provocada pela desuniformidade entre os emissores. Para Solomon (1979) é, também, uma informação que expressa bem o projeto do emissor, materiais usados na construção e cuidados aplicados na sua fabricação. De acordo com Abreu et al. (1987) por mais sofisticados que sejam os processos de fabricação, é impossível obter-se emissores com o mesmo coeficiente $k_{d} \mathrm{e}$ expoente $x$ da função potencial. Os autores acrescentam, ainda, que dentre os diversos tipos de emissores desenvolvidos, os desmontáveis e/ou auto-compensantes apresentam excelentes coeficientes de variação de fabricação.

Solomon (1979) estudando as variações da fabricação de vários emissores tipo orifício, classificou e deu uma interpretação física para o coeficiente de variação de fabricação (CVF) relacionando o desvio-padrão da vazão dos emissores com a vazão média da amostra submetida a testes com pressão constante. Para análise do CVF, a ABNT (1986) recomenda uma amostragem aleatória de 20 a 50 emissores, respectivamente no caso de microaspersores e gotejadores. Segundo a ASAE (1994) os critérios de classificação dos emissores, em função do CVF, variam de 0,05 a 0,15 , considerando-se valores abaixo de 0,05 excelentes e, acima de 0,15 , inaceitáveis.
Estudando as características hidráulicas de tubos perfurados flexíveis de polietileno, Bonomo et al. (1998) verificaram alcance médio da precipitação de 3,02, 4,02, 4,45 e 4,78 m para as pressões de 20,40,60 e $80 \mathrm{kPa}$, respectivamente. Andrade (1990) encontrou os seguintes valores médios de alcance: 2,87, 4,10, 5,10 e 5,92 m para as mesmas pressões de serviço. Quanto ao CVF, Andrade (1990) e Bonomo et al. (1998) verificaram que o coeficiente de variação médio foi de 1,95 e $3,31 \%$, respectivamente, o que classifica os emissores como excelentes. Testezlaf \& Campioni (1993) encontraram CVF médio de 2,8\% para o tubo gotejador Queen Gil, com emissores tipo labirinto, espaçados $0,30 \mathrm{~m}$, indicando boa qualidade de fabricação; já Frizzone et al. (1998) encontraram, para o tubo gotejador Rain-Tape TPC, CVF médio de 1,97\%, valor este inferior ao informado pelo fabricante.

O objetivo deste trabalho foi determinar as características hidráulicas do Santape, analisando-se a equação característica do emissor, o coeficiente de variação de fabricação, o diâmetro interno em função da pressão e a pressão de ruptura da parede do tubo, alcance e altura do jato da água e a vazão em função do aumento do comprimento e da pressão.

\section{MATERIAL E MÉTODOS}

Este trabalho foi realizado no Laboratório de Engenharia de Irrigação e Drenagem do Departamento de Engenharia Agrícola do Centro de Ciências e Tecnologia da Universidade Federal da Paraíba (LEID/DEAg/CCT/UFPB) na cidade de Campina Grande, e nele foi estudada a performance hidráulica do Santape fabricado pela Santeno Irrigações do Nordeste Ltda., produzido a partir de tecnologia japonesa da Sumitomo Chemical; trata-se de tubo perfurado flexível de polietileno, fabricado com polietileno linear de baixa densidade, a partir de matéria-prima virgem, com diâmetro de $28 \mathrm{~mm}$ e espessura da parede de $0,24 \mathrm{~mm}$. O emissor consiste de um conjunto de dois orifícios, cujo diâmetro é de $0,3 \mathrm{~mm}$ cada um, perfurado a raio laser. $\mathrm{O}$ espaçamento entre emissores varia de 0,15 a 1,20 m, com comprimento de até $200 \mathrm{~m}$ e trabalha com uma pressão de serviço de 20 a $80 \mathrm{kPa}$. A Santeno produz dois tipos de tape: o Tipo I, com os microfuros no centro do tape, resultando num raio de alcance de até $2,5 \mathrm{~m}$ e altura do jato d'água de até 2,0 m e o Tipo II, com microfuros na lateral do tape, raio de alcance de até 2,0 m e altura do jato d'água de até 0,5 m. Em ambos os casos, a água é aspergida para os dois lados em jatos bem finos, em forma de spray, formando uma faixa molhada. A vazão do tape varia de 2,82 a 30,50 $\mathrm{L} \mathrm{h}^{-1} \mathrm{~m}^{-1}$. Foram efetuados testes com os Santapes I e II, com orifícios espaçados $1,20 \mathrm{~m}$.

Para avaliação dos orifícios, a vazão foi determinada pelo método gravimétrico utilizando-se, como coletores, recipientes cilíndricos de PVC, com capacidade para $1076 \mathrm{~mL}$; a pesagem dos recipientes foi feita em balança eletrônica, com precisão de $0,1 \mathrm{~g}$ e os testes executados de acordo com as normas da ABNT (1986) cujo sistema foi montado sobre tijolos a uma altura de $20 \mathrm{~cm}$ acima do solo e de maneira invertida, de modo a facilitar a coleta dos volumes. Foram utilizados quatro segmentos de $11 \mathrm{~m}$ de Santape II, escolhidos e cortados aleatoriamente, totalizando 36 emissores (pares de orifícios). A duração para cada coleta foi de 5 min, tempo suficiente para se coletar volume superior a 
$200 \mathrm{~mL}$, cronometrados com precisão de décimos de segundos; após a coleta, os recipientes foram pesados em balança eletrônica com precisão de $0,1 \mathrm{~g}$ e, em seguida, os dados foram transformados em unidades de volume. A vazão de cada emissor foi obtida calculando-se a média aritmética das três repetições e, por meio de análise de regressão, ajustou-se a equação característica do emissor.

Os emissores foram submetidos às pressões de 20,40,60 e $80 \mathrm{kPa}$, recomendadas pelo fabricante, obtidas e reguladas por meio de registros manuais e lidas através de manômetro de mercúrio.

O CVF dos emissores foi obtido através dos valores da média das vazões dos emissores e desvio-padrão da vazão para cada pressão recomendada pelo fabricante, conforme equação proposta por Keller \& Bliesner (1990). A partir dos valores obtidos do coeficiente de variação, foi possível se classificar os emissores segundo a uniformidade de fabricação, de acordo com a classificação proposta pela ASAE (1994).

Em virtude de ser o Santape flexível, com o aumento da pressão ocorre também aumento no seu diâmetro. Para se determinar o diâmetro interno e a pressão de ruptura, foi escolhido, aleatoriamente, um rolo de Santape II com furos e, deste, cortados três segmentos de $5,0 \mathrm{~m}$. O sistema foi montado no laboratório e para cada pressão de serviço fez-se a medição do diâmetro externo através de um paquímetro; descontando-se a espessura da parede $(0,24 \mathrm{~mm})$ obteve-se o diâmetro interno.

A pressão de ruptura da parede do tubo foi determinada aumentando-se sua pressão interna através de uma regulagem lenta e gradual no registro de gaveta e lida no manômetro de mercúrio, sendo possível visualizar o momento da ruptura do tubo. Para se determinar o alcance e a altura do jato d'água foram realizados ensaios em laboratório, sem interferência do vento, seguindo as normas propostas por Merriam \& Keller (1978) para tubulações perfuradas e as normas da ABNT (1986).

Foram montados, a nível do solo, dois sistemas com Santape I e Santape II, ambos com 6,20 m de comprimento, instalados entre quatro linhas de coletores, espaçados uniformemente de $20^{\prime} 20 \mathrm{~cm}$ e distanciados $3,20 \mathrm{~m}$, totalizando 120 coletores. O tempo de funcionamento de cada ensaio foi de uma hora, com três repetições para cada pressão estudada.

A pressão foi regulada através de registro de gaveta e lida por um manômetro de mercúrio. Para cada pressão foram feitas leituras de volume de água contido em cada coletor através do método da pesagem, em balança eletrônica com precisão de $0,1 \mathrm{~g}$; em seguida, as leituras de volume foram convertidas em altura de precipitação.

Na determinação do alcance da precipitação utilizou-se a norma proposta pela ABNT (1986) a qual diz que a precipitação mínima para se definir o alcance do emissor deve ser correspondente a $0,13 \mathrm{~mm} \mathrm{~h}^{-1}$ para emissores com vazão inferior a $0,50 \mathrm{~m}^{3} \mathrm{~h}^{-1}$, podendo ser obtido por interpolação.

A altura do jato d'água foi determinada, para as pressões de 20, 40, 60 e $80 \mathrm{kPa}$, nas distâncias pré-determinadas de $0,8,1,6$, 2,4 e 3,2 m para o Santape II e, nas distâncias de 0,75, 1,50, 2,25 e 3,0 m, para o Santape I. Após a pressão se estabilizar, as leituras das alturas foram feitas com uma régua graduada de metal, em três pontos de emissão diferentes, com três repetições.
A vazão foi avaliada nos comprimentos de 10,30, 60, 90, 120, 150,180 e $200 \mathrm{~m}$, e nas pressões de 20, 40, 60 e $80 \mathrm{kPa}$, com espaçamento de 1,20 m entre emissores. Foram coletados volumes em seis pontos de emissão ao longo do comprimento, sendo estes múltiplos do espaçamento entre emissores, e se considerando, sempre, o primeiro e o último emissor. A vazão por unidade de comprimento foi obtida multiplicando-se a vazão média por emissor (composto de um par de orifícios) pelo número total de emissores na linha e dividindo-se pelo comprimento da tubulação.

\section{RESULTADOS E DISCUSSÃO}

A partir da análise de regressão dos dados de vazão versus pressão, obteve-se a equação $\mathrm{q}=0,43 \mathrm{H}^{0,56}$, onde q é a vazão do emissor em $\mathrm{L} \mathrm{h}^{-1}$ para o conjunto de 2 orifícios e $\mathrm{H}$ é a pressão de entrada do emissor, em $\mathrm{kPa}$. O coeficiente de determinação $\left(\mathrm{r}^{2}\right)$ da equação foi de 0,99 , indicando um bom ajuste do modelo potencial para descrever a relação vazão-pressão. Dentro da faixa de pressão em que esta equação é válida, os orifícios operam em regime de escoamento turbulento e seu expoente ficou próximo a 0,5 , como se esperava. A representação gráfica da equação obtida encontra-se na Figura 1.

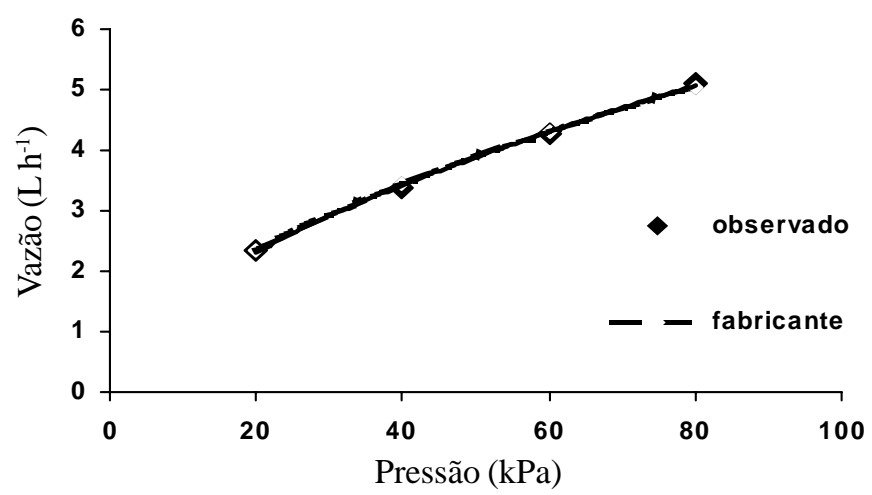

Figura 1. Curvas da relação vazão-pressão dos emissores do Santape II

O catálogo do fabricante apresenta a equação potencial do emissor com o valor de $k_{d}=0,45$ e $x=0,55$, operando com a unidade de pressão em mca. Observa-se, na Figura 1, que a curva da relação vazão-pressão do fabricante e a obtida neste trabalho se sobrepõem. Andrade (1990) trabalhando com unidade de pressão em mca e Bonomo et al. (1998) na unidade de kPa, encontraram valores de $k_{d}$ iguais a 1,6085 e 0,4748 e de $x, 0,5491$ e 0,5 , respectivamente, para tubos perfurados de polietileno flexível, indicando o regime de escoamento como turbulento. Esses valores se aproximaram dos obtidos neste trabalho e, para o tubo gotejador Queen Gil, Testezlaf \& Campioni (1993) encontraram valor de $x$ igual a 0,625. Frizzone et al. (1998) avaliando as características hidráulicas do tubo gotejador Rain-Tape TPC, obtiveram valor de $x$ como 0,4563 . Os dois tipos de tubo gotejador foram caracterizados como de escoamento turbulento.

Os CVF variaram de 3,73 a 4,50\% para as pressões de 20,40, 60 e $80 \mathrm{kPa}$, com média de 4,10\%; esses resultados estão apresentados na Tabela 1. De acordo com a classificação proposta pela ASAE em todas as faixas de pressão estudadas, 
os emissores utilizados podem ser classificados como excelentes, em termos de uniformidade de fabricação, comprovando a uniformidade na perfuração dos orifícios com raio laser.

Tabela 1. Vazão média em função da pressão para os emissores do Santape II, com seus respectivos valores de coeficiente de variação de fabricação (CVF)

\begin{tabular}{ccc}
\hline Pressão $(\mathrm{kPa})$ & Vazão Média $\left(\mathrm{L} \mathrm{h}^{-1}\right)$ & $\mathrm{CVF}(\%)$ \\
\hline 20 & 2,33 & 3,73 \\
40 & 3,39 & 4,29 \\
60 & 4,27 & 4,50 \\
80 & 5,12 & 3,90 \\
\hline Média & - & 4,10 \\
\hline
\end{tabular}

Valores semelhantes também foram encontrados por Andrade (1990) e Bonomo et al. (1998) estudando as características hidráulicas de um tubo perfurado de polietileno flexível, tendo observado coeficientes médios de variação de 1,95 e 3,31\%, respectivamente, o que classifica os emissores como excelentes.

Os resultados das medições do diâmetro interno estão apresentados na Tabela 2 e, como se pode observar, os diâmetros internos aumentam com o incremento da pressão; isto ocorre em virtude da tubulação ser flexível; este aumento também foi verificado por Andrade (1990), ao estudar tubos perfurados de polietileno flexível, e por Frizzone et al. (1998), ao determinar as características hidráulicas do tubo gotejador Rain-Tape. O fabricante do tape apresenta valores bem próximo aos obtidos neste trabalho.

Tabela 2. Valores dos diâmetros internos do Santape II observados para diferentes pressões

\begin{tabular}{cc}
\hline Pressão $(\mathrm{kPa})$ & Diâmetro Interno $(\mathrm{mm})$ \\
\hline 20 & 27,82 \\
40 & 28,22 \\
60 & 28,32 \\
80 & 29,02 \\
\hline
\end{tabular}

A pressão de ruptura média da parede do tubo foi de $169,30 \mathrm{kPa}$, cujo valor está dentro do intervalo indicado pelo fabricante. Testezlaf \& Campioni (1993) determinaram a pressão média de ruptura da parede de polietileno do tubo gotejador Queen Gil, a qual foi igual a 383,50 kPa; eles observaram, também, que o ponto em que mais freqüentemente a tubulação se rompeu foi sobre a dobra do tubo gotejador, onde se localizava o início ou a entrada do labirinto. Frizzone et al. (1998) obtiveram, para o tubo gotejador Rain-Tape TPC, uma pressão de ruptura em torno de 206,0 kPa e observaram que, a partir de $166,80 \mathrm{kPa}$, o tubo começou a deformar-se ao longo dos $5 \mathrm{~m}$ e o processo de ruptura sempre ocorreu na parede do tubo e não nos locais onde são posicionados os emissores.

$\mathrm{O}$ alcance do jato de água foi determinado a partir dos dados de precipitação, em função da pressão e da posição dos orifícios na tubulação. Os valores estão apresentados na Tabela 3. Os perfis de distribuição obtidos indicam aumento no alcance do jato, em função do incremento na pressão, para os Santapes I e II, cujo alcance mínimo foi de 1,30 e 1,70 m e o máximo de 2,50 e 3,00 m, para as pressões de 20 e $80 \mathrm{kPa}$, respectivamente. Observa-se que a precipitação começa a partir dos $60 \mathrm{~cm}$ de distância perpendicular, aumentando com a pressão; isto significa que, no campo, a lateral deve ser instalada no meio da linha de plantio ou se fazendo sobreposição das linhas.

Tabela 3. Valores do alcance médio do jato d'água nos Santapes I e II, em função da pressão

\begin{tabular}{ccc}
\hline $\begin{array}{c}\text { Pressão } \\
(\mathrm{kPa})\end{array}$ & \multicolumn{2}{c}{ Alcance Médio do Jato $(\mathrm{m})$} \\
\cline { 2 - 3 } & Santape I & Santape II \\
\hline 20 & 1,30 & 1,70 \\
40 & 2,00 & 2,50 \\
60 & 2,30 & 2,70 \\
80 & 2,50 & 3,00 \\
\hline
\end{tabular}

Os valores obtidos do alcance médio foram superiores aos indicados pelo fabricante, ou seja, 2,0 m para o Santape II e 2,5 m para o Santape I, enquanto Bonomo et al. (1998) estudando o Santape I, obtiveram alcance médio de 2,39 m e Andrade (1990) encontrou um valor médio de 2,96 m no Santape II, ambos operando com a pressão de $80 \mathrm{kPa}$.

A altura do jato de água foi determinada para os Santape I e II, ao longo do raio de alcance, dividindo-se este em três pontos eqüidistantes. Os valores obtidos encontram-se na Tabela 4. Observou-se que, para o Santape II até 1,60 m de distância do emissor, a água é aspergida em duas alturas

Tabela 4. Valores de altura do jato de água em função da pressão e da distância para os Santape I e II

\begin{tabular}{|c|c|c|c|c|c|c|c|c|}
\hline \multirow{2}{*}{$\begin{array}{l}\text { Distância } \\
\text { (m) }\end{array}$} & \multicolumn{2}{|c|}{$\mathrm{P}=20 \mathrm{kPa}$} & \multicolumn{2}{|c|}{$\mathrm{P}=40 \mathrm{kPa}$} & \multicolumn{2}{|c|}{$\mathrm{P}=60 \mathrm{kPa}$} & \multicolumn{2}{|c|}{$\mathrm{P}=80 \mathrm{kPa}$} \\
\hline & $\mathrm{H}_{1}$ & $\mathrm{H}_{2}$ & $\mathrm{H}_{1}$ & $\mathrm{H}_{2}$ & $\mathrm{H}_{1}$ & $\mathrm{H}_{2}$ & $\mathrm{H}_{1}$ & $\mathrm{H}_{2}$ \\
\hline 0,75 & - & 0,77 & 0,75 & $\begin{array}{r}\text { APE I } \\
1,32\end{array}$ & 0,90 & 1,43 & 1,01 & 1,80 \\
\hline 1,50 & - & - & - & 1,0 & - & $1, .38$ & - & 1,63 \\
\hline 2,25 & - & - & - & - & - & 0,71 & - & 1,20 \\
\hline 0,80 & 0,24 & 0,53 & 0,32 & $\begin{array}{c}\text { APE II } \\
0,67\end{array}$ & 0,35 & 0,68 & 0,37 & 0,72 \\
\hline 1,60 & - & 0,16 & 0,32 & 0,80 & 0,52 & 1,10 & 0,61 & 1,24 \\
\hline 2,40 & - & - & - & 0,44 & - & 0,84 & - & 1,06 \\
\hline
\end{tabular}


diferentes e, depois, o jato é pulverizado, sendo possível medir apenas uma altura. No Santape I, o jato se pulveriza a uma distância menor, a 1,50 m; essas diferenças ocorrem devido a posição dos furos nos tapes serem diferentes.

Valores de vazão, por pares de orifícios, em função da pressão e do comprimento, foram obtidos experimentalmente e estão apresentados na Tabela 5. Observa-se que os valores de vazão aumentam com a pressão, porém diminuem com o comprimento do tape. Comparando-se esses dados com os que o fabricante apresenta no catálogo observou-se que, para o mesmo espaçamento entre orifícios de $1,05 \mathrm{~m}$ e comprimento de $200 \mathrm{~m}$, os valores de vazão obtidos foram superiores aos fornecidos pelo fabricante, cuja diferença maior ocorreu para as pressões de 20 e $40 \mathrm{kPa}$, correspondendo a 19,66 e $14,11 \%$, respectivamente, mas para as pressões de 60 e $80 \mathrm{kPa}$ esta diferença diminuiu para 6,05 e $1,84 \%$, respectivamente.

Tabela 5. Valores de vazão $\left(\mathrm{L} \mathrm{h}^{-1} \mathrm{~m}^{-1}\right)$ em função da pressão e do comprimento do Santape, obtidos experimentalmente

\begin{tabular}{ccccccccc}
\hline $\begin{array}{c}\text { Pressão } \\
(\mathrm{kPa})\end{array}$ & 10 & 30 & 60 & 90 & 120 & 150 & 180 & 200 \\
\cline { 2 - 8 } & 4,71 & 3,68 & 3,86 & 3,70 & 3,84 & 3,79 & 3,87 & 3,51 \\
20 & 6,78 & 5,36 & 5,51 & 5,22 & 5,47 & 5,23 & 5,46 & 4,89 \\
40 & 8,61 & 6,67 & 6,78 & 6,65 & 6,77 & 6,62 & 6,62 & 5,95 \\
60 & 11,25 & 8,09 & 8,48 & 8,05 & 8,10 & 7,98 & 7,72 & 7,09 \\
80 & 11,25 &
\end{tabular}

Para determinação das vazões em diferentes pressões e comprimentos não testados, foram ajustadas equações matemáticas polinomiais de terceiro grau a esses pontos. As equações encontradas para as respectivas pressões estão apresentadas na Tabela 6 , juntamente com os coeficientes de determinação. Para as pressões de 20 e 40 $\mathrm{kPa}$, a equação de regressão não foi significativa; já nas pressões de 60 e $80 \mathrm{kPa}$, o ajuste foi significativo a nível de $1 \%$ de probabilidade.

A Figura 2 apresenta os valores de vazão, estimados a partir das equações da Tabela 6 , onde se observa que, para as pressões de 20,40 e $60 \mathrm{kPa}$, a curva se apresenta quase linear, ocorrendo pouca variação da vazão ao longo da tubulação, o que mostra a boa uniformidade de vazão ao longo do comprimento. Na pressão de $80 \mathrm{kPa}$ a curva se apresenta com maior ondulação, ocorrendo maior variação da vazão. Observa-se que os valores estimados foram bem próximo aos obtidos experimentalmente, sendo que as maiores diferenças ocorreram para as pressões de 40 e $80 \mathrm{kPa}$; isto pode ter ocorrido em virtude da precisão do ajuste da equação ter sido menor.

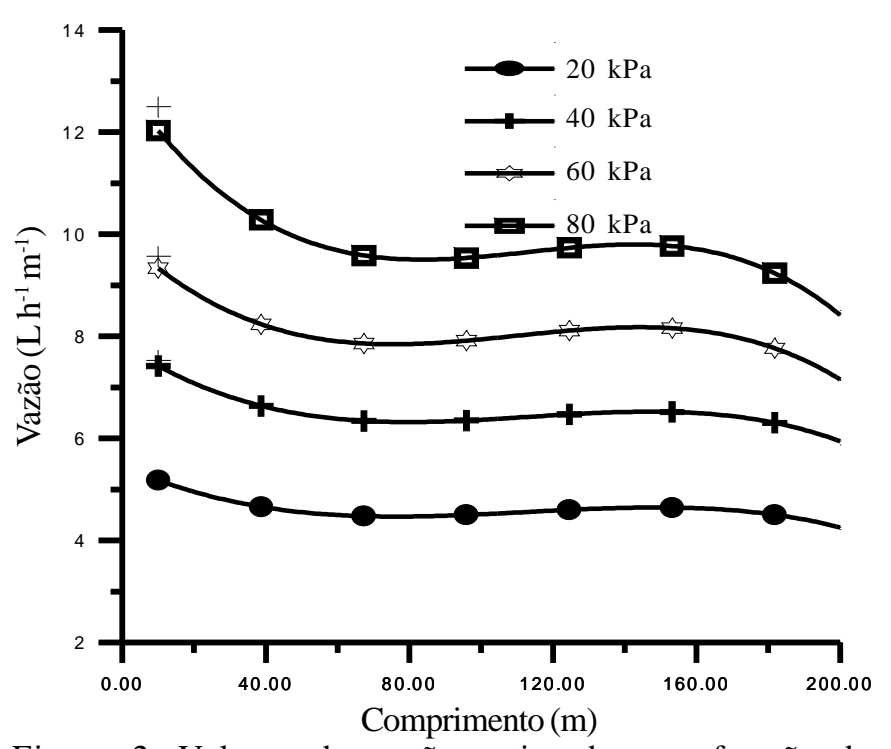

Figura 2. Valores de vazão estimados em função do comprimento da lateral com Santape nas pressões de 20, 40, 60 e $80 \mathrm{kPa}$

\section{CONCLUSÕES}

1. A equação característica dos emissores do Tape Santape ajustou-se a um modelo potencial, em que o expoente foi igual a 0,56 , indicando que os emissores operam em regime de escoamento turbulento.

2. O coeficiente de variação de fabricação dos emissores foi classificado como excelente, conforme a norma americana ASAE (1994); isto comprova a uniformidade na perfuração dos orifícios feitos a raio laser.

3. O diâmetro interno do Santape aumentou com incrementos na pressão e sua parede se rompeu à pressão de $169,30 \mathrm{kPa}$.

4. Os alcances do jato de água variaram em função da pressão, sendo maiores para o Santape II; já as alturas do jato de água encontradas para o Santape I foram maiores que para o Santape II;

5. Os valores de vazão obtidos para o comprimento de 200 $\mathrm{m}$, foram superiores aos divulgados pelo fabricante.

\section{REFERÊNCIAS BIBLIOGRÁFICAS}

ABREU, J.M.H.; LOPES, J.R.; REGALADO. A.P.; HERNANDEZ, J.F.G. El riego localizado. Madrid: Instituto Nacional de Investigaciones Agrárias, 1987.317p. AMERICAN SOCIETY OF AGRICULTURAL ENGINEERS - ASAE. Design and installation of microirrigation systems. St: Joseph, 1994. 4p. EP405.1 DEC94

Tabela 7. Equações polinomiais da vazão em função do comprimento e da pressão com os respectivos coeficientes de determinação $\left(\mathrm{r}^{2}\right)$

\begin{tabular}{ccc}
\hline $\begin{array}{c}\text { Pressão } \\
(\mathrm{kPa})\end{array}$ & Equação de Regressão & $\mathrm{r}^{2}$ \\
\hline 20 & $\mathrm{Q}=4,969+0,039 \mathrm{~L}+3,797 \times 10^{-4} \mathrm{~L}^{2}+1,091 \times 10^{-6} \mathrm{~L}^{3}$ & $0,893^{\mathrm{ns}}$ \\
40 & $\mathrm{Q}=7,097+0,058 \mathrm{~L}+5,594 \times 10^{-4} \mathrm{~L}^{2}+1,610 \times 10^{-6} \mathrm{~L}^{3}$ & $0,814^{\mathrm{ns}}$ \\
60 & $\mathrm{Q}=9,035-0,080 \mathrm{~L}+7,862 \times 10^{-4} \mathrm{~L}^{2}-2,311 \times 10^{-6} \mathrm{~L}^{3}$ & $0,853^{* *}$ \\
80 & $\mathrm{Q}=11,777+0,117 \mathrm{~L}+1,098 \times 10^{-3} \mathrm{~L}^{2}+3,162 \times 10^{-6} \mathrm{~L}^{3}$ & $0,833^{* *}$ \\
\hline
\end{tabular}

* $\mathrm{Q}=$ vazão $\mathrm{em} \mathrm{L} \mathrm{h}^{-1} \mathrm{~m}^{-1} ; \mathrm{L}=$ comprimento em $\mathrm{m}$

ns não significativo

** significativo a nível de 5\% de probabilidade 
ANDRADE, D.V. Avaliação hidráulica de tubos flexíveis de polietileno perfurados a laser utilizados na irrigação. Piracicaba: ESALQ, 1990, 147p. Dissertação de Mestrado

ASSOCIAÇÃO BRASILEIRA DE NORMAS TÉCNICAS - ABNT. Rio de Janeiro, RJ). Emissores para irrigação localizada: Avaliação de características operacionais. Rio de Janeiro, 1986. 6p. Projeto 12:02.08.21

BONOMO, R.; GOMES FILHO, R.R.; MANTOVANI, E.C.; RAMOS, M.M. Caracterização hidráulica de tubos perfurados flexíveis de polietileno. Engenharia Agrícola, Jaboticabal, v.17, n.3, p.60-69, 1998.

BRALTS, V.F.; WU, I.P.; GITLIN, H.M. Manufacturing variation and drip irrigation uniformity. Transactions of the ASAE. St. Joseph, v. 24, n.1, p. 113-119, 1981.

FRIZZONE, J.A.; VIEIRA, A.T.; PAZ, V.P da S.; BROTEL, T.A. Caracterização hidráulica de um tubo gotejador. Revista Brasileira de Engenharia Agrícola e Ambiental. Campina Grande, v.2,n.3: p.278-283, 1998.
KELLER, J.; BLIESNER, R.D. Sprinkle and trickle irrigation . New York: van Nodtrand Reinhold, 1990. 652p.

LÒPEZ, J.R; ABREU, J.M.H.; REGALADO, A.P.; HERNÁNDEZ, J.F.G. Riego localizado. Madrid: Mundi-Prensa, 405 p. 1992.

MERRIAM, J.L.; KELLER, J. Trickle irrigation design. Riverside: Rain Bird Sprinkler Manufacturing Corporation, $1978.133 \mathrm{p}$

SOLOMON, K.H. Manufacturing variation of trickle emitters. Transactions of the ASAE. St. Joseph, v. 22, n.4, p.1034-1038, 1979.

TESTEZLAF, R.; CAMPIONI, E. Comportamento hidráulico do tubo gotejador "Queen Gil". Engenharia Agrícola. Campinas, v.13, p.29-38, 1993.

VERMEIREN, I.; JOBLING, G. A. Localized irrigation. Rome: FAO, 1980. 203p. Irrigation and Drainage Paper, 36

von BERNUTH, R.; SOLOMON, K.H. Emitter construction. In: Nakayama, F.S.; Bucks, D.A Trickle irrigation for crop production. Phoenix: Elsevier 1986. cap. 2, p. 27-52. 